\title{
Martina Schmidhuber (2020) Ein gutes Leben für Menschen mit Demenz: Ethische Herausforderungen in Betreuung und Pflege (Ethik der Lebenswelten, Band 6; Hrsg.: Peter Kampits)
}

\author{
LIT Verlag, Wien, 133 Seiten, 29,90€, ISBN 978-3-643-50986-4
}

\section{Henrike Voß}

Angenommen: 10. Februar 2021 / Online publiziert: 16. Februar 2021

(C) Der/die Autor(en) 2021

Einer grundlegenden ethischen Perspektive geht Martina Schmidhuber, Philosophin, Ethikerin und Medizintheoretikerin, in ihrem Buch nach - der Frage nach dem guten Leben für Menschen mit Demenz.

Wenn in unserer Gesellschaft Rationalität und Bewusstsein als menschliche Grundkompetenzen gelten, stellt sich die Frage, woran wir im Zusammenhang von Demenz ein gutes Leben festmachen können und welche Aufgaben auf Angehörige und im Gesundheitswesen Tätige zukommen. Die Autorin fokussiert sich auf Personen mit einer Alzheimer Demenz und führt in neun Themenkapiteln aus, welche Herausforderungen sich etwa einer Pflegedyade stellen (können). Schmidhuber nimmt sowohl individuelle Betreuungssituationen als auch gesamtgesellschaftliche Themen in den Blick. So soll das Buch als Orientierungshilfe in ethisch herausfordernden Situationen mit Menschen mit Demenz dienen und möchte zum Weiterdenken anregen (S. 2).

Schmidhuber legt in Kapitel 1 die Grundlagen zur Alzheimer-Demenz dar. Ganz im Kitwood'schen Sinne verweist die Autorin immer wieder darauf, dass Menschen mit Demenz eine Stimme verliehen und sie nicht segregiert und pathologisiert werden sollten. Sie stellt die zunehmende Abhängigkeit der erkrankten Personen vom unterstützenden Umfeld heraus - als Sekundärbetroffene nehmen An- und Zugehörige immer wieder eine zentrale Rolle in den Schilderungen ein. In Kapitel 2 werden die Vor- und Nachteile einer frühen Demenzdiagnose thematisiert. Unter anderem verweist der Text darauf, dass das Wissen, erkrankt zu sein, manchen Personen mehr schaden als nutzen kann. Weiterhin geht die Autorin auf die hohe Bedeutung der „Vorab-Planung“ ein, des Advance Care Planning (ACP) als wichtigem Mittel zur Selbstbestimmung (S. 13). Sie weist auf die generellen Antizipationsprobleme in der

H. Voß, M.A. (殴

Ruprecht-Karls-Universität Heidelberg, Heidelberg, Deutschland

E-Mail: voss@nar.uni-heidelberg.de 
Vorausplanung hin und zeigt auf, dass diese gerade im Fall von Demenz besonders relevant werden, weil das Prinzip der informierten Entscheidungen hier an Grenzen stößt (S. 15).

In den Kapiteln 3 und 4 wird zunächst das Autonomie-Verständnis in der philosophischen Debatte dargelegt. Im Kontext von Demenz plädiert die Autorin dafür, diesen Begriff graduell zu verstehen, und knüpft eng an das Konzept von Relationalität an, denn ,ein gutes Leben für Menschen mit Demenz [ist] ohne die Unterstützung anderer Menschen nicht möglich“ (S. 34). Sie fragt, ob nicht besser von ,Unterstützung“ denn von relationaler Autonomie gesprochen werden sollte (S. 36). Weiter wird in diesem Zusammenhang der Personenstatus auf seine Praxistauglichkeit untersucht. Hier wägt sie die Leiblichkeit und Emotionalität als bisher vernachlässigte Kriterien von Personalität gegen die Bedeutung von Erinnerung und Rationalität für den Personenstatus ab.

Ausgehend von ,einem sehr praktischen, lebensnahen Verständnis personaler Identität“ (S. 49) fokussiert sich Schmidhuber hierbei in Kapitel 5 auf Patientenverfügungen und fragt, ,inwiefern eine Demenz personale Identität beeinflusst“ (S. 50). So gehe das Bewusstsein beim Menschen mit Demenz nicht verloren, sondern verändere sich (S. 56). Es sei Aufgabe der Angehörigen, die Identität der Person fortzuführen, indem die Lebensgeschichte weitererzählt wird. Kapitel 6 beleuchtet interkulturelle Aspekte im Umgang mit Betroffenen, wobei etwa das Prinzip der Selbstbestimmung, Sprache und Kommunikation, Informed Consent, Fremdheit und Identität oder interkulturelle Kompetenz Erwähnung finden.

Im nachfolgenden Kapitel 7 plädiert die Autorin dafür, ,eine neue, andere Perspektive einzunehmen, die zeigt, dass auch Menschen mit Alzheimer in manchen Phasen der Erkrankung Lebensqualität empfinden können“, jedoch stets unter der Prämisse, dass Lebensqualität ,ein vages und komplexes Konzept ist“ (S. 78). So veranschaulicht sie anhand von Narrationen, dass die subjektive Lebensqualität im Vordergrund stehen sollte. In Kapitel 8 untersucht Schmidhuber den (Stellen-)Wert der Wahrheit in der Situation von Demenz. Die subjektive Wahrheitswahrnehmung eines Betroffenen verändert sich und so fragt die Autorin: Darf das betreuende Umfeld lügen oder täuschen mit dem Ziel, den Alltag des Betroffenen zu verbessern? Der Text macht hier - auch anhand von Beispielen - deutlich, dass als oberstes Gebot stets die Orientierung an der subjektiven Wirklichkeit des Menschen mit Demenz gelten sollte und diese etwa ,bei der Validation als auch bei der Scheinweltgestaltung im Mittelpunkt" stehen sollte (S. 99). Kapitel 9 widmet sich dem menschenwürdigen Sterben mit Demenz. Der Begriff der Würde wird hier in seinem Facettenreichtum dargelegt. Schmidhuber diskutiert Themen der künstlichen Ernährung oder des freiwilligen Verzichts auf Flüssigkeit und Nahrung (FVFN). Sie fordert mit Blick auf Letzteres, dass ,,es ethisch geboten [sei], diesen Wunsch ernst zu nehmen und im Sinne der Sterbebegleitung diese Menschen bei ihrem für sie würdigen Abschied aus dem Leben zu begleiten“ (S. 116).

„Ethik fängt bei der Sprache an“ (S. 2): diesem Credo wird die Autorin durch die Auswahl der Buchthemen in jedem Fall gerecht - sie rückt stetig die Person mit Demenz als soziales Wesen in den Mittelpunkt. Das als Informationsquelle anhand von Überlegungen mit Anleitungen vornehmlich für praktisch Tätige (S. 2) konzipierte Buch behandelt eine Vielfalt demenzspezifischer Themen. Philosophi- 
sche Grundbegriffe werden stets eingeführt, bevor deren Anwendbarkeit auf das gute Leben für Menschen mit Demenz überprüft wird. Tiefergehende Ausführungen wären an einigen Stellen hilfreich, da Konzepte, etwa wie ACP oder Advance Care Dialogue (ACD), in der Praxis vielfach noch unbekannt sind. An einigen Stellen irritieren zu absolut formulierte Aussagen - etwa: ,[...], weil Menschen mit Demenz ihre Zukunft nicht mehr antizipieren können [...]“ (S. 80) oder „Eine Spezialstation mit Tapetentüren [...] [ist] zu unterlassen.“ (S. 101) - und verursachen ein sicherlich ungewolltes Bild von Ausschließlichkeit. Dessen ungeachtet gelingt es dem Buch, deutlich zu machen, dass eine Unterstützung eines guten Lebens von Menschen mit Demenz ein erstrebenswertes Ziel ist. In jedem Fall erhalten ,,alle in diesem Umfeld Tätigen“ (S. ii) einen guten Überblick über das Themenspektrum für die Praxis.

Funding Open Access funding enabled and organized by Projekt DEAL.

Open Access Dieser Artikel wird unter der Creative Commons Namensnennung 4.0 International Lizenz veröffentlicht, welche die Nutzung, Vervielfältigung, Bearbeitung, Verbreitung und Wiedergabe in jeglichem Medium und Format erlaubt, sofern Sie den/die ursprünglichen Autor(en) und die Quelle ordnungsgemäß nennen, einen Link zur Creative Commons Lizenz beifügen und angeben, ob Änderungen vorgenommen wurden.

Die in diesem Artikel enthaltenen Bilder und sonstiges Drittmaterial unterliegen ebenfalls der genannten Creative Commons Lizenz, sofern sich aus der Abbildungslegende nichts anderes ergibt. Sofern das betreffende Material nicht unter der genannten Creative Commons Lizenz steht und die betreffende Handlung nicht nach gesetzlichen Vorschriften erlaubt ist, ist für die oben aufgeführten Weiterverwendungen des Materials die Einwilligung des jeweiligen Rechteinhabers einzuholen.

Weitere Details zur Lizenz entnehmen Sie bitte der Lizenzinformation auf http://creativecommons.org/ licenses/by/4.0/deed.de. 